\title{
Near Total Transection of the Obturator Nerve and Primary Repair during Laparoscopic Radical Hysterectomy for Cervical Cancer
}

\author{
Yong Kuei Lim* \\ Department of Gynaecologic Oncology, KK Women's \& Children's Hospital, Singapore \\ *Corresponding author: Timothy.lim.yk@kkh.com.sg
}

\begin{abstract}
Background: Obturator nerve injury is a rare complication and it may occur during pelvic cancer surgery especially during pelvic lymphadenectomy. The injury may cause paraesthesia, sensory loss or pain in the medial thigh which may extend down to the knee. It can also cause weakness in adduction with gait disturbance, medial thigh wasting and possible medico-legal consequences. Case Report: The patient is a 57 year old lady who was found to have an early cervical cancer. MRI abdomen and pelvis was negative for regional lymphadenopathy or distant metastases hence clinically she had a Stage IB1 SCC of cervix. She underwent a Total Laparoscopic Wertheim's Radical Hysterectomy BSO Bilateral Pelvic Lymphadenectomy in February 2015. During the pelvic lymphadenectomy, the left obturator nerve was inadvertently damaged and partially transected by the Harmonic scalpel. The nerve was reconstituted by interrupted 5-O vicryl suture to the epineural layer of the nerve. Post operatively, the patient experienced weakness of the adductor muscles (3/5) but negligible sensory loss. Four months after intensive physiotherapy, the motor deficit was markedly reduced and her gait was normal. There was no sensory loss. Final histology revealed that the surgical margins was clear and $0 / 41$ pelvic lymph nodes were negative. Hence she did not need any adjuvant therapy. Conclusion: Obturator nerve injury is a rare complication with a significant clinical impact if unrecognised. In uncomplicated cases, laparoscopic route of repair is feasible and safe and an immediate repair of the nerve is preferable.
\end{abstract}

Keywords: cervical cancer, obturator nerve injury, laparoscopy

Cite This Article: Yong Kuei Lim, "Near Total Transection of the Obturator Nerve and Primary Repair during Laparoscopic Radical Hysterectomy for Cervical Cancer." American Journal of Medical Case Reports, vol. 5, no. 7 (2017): 174-176. doi: 10.12691/ajmcr-5-7-1.

\section{Introduction}

Obturator nerve injury is an uncommon complication that may occur during pelvic cancer surgery especially during pelvic lymphadenectomy. The injury usually results from sectioning, stretching or crushing of the nerve. [1] The obturator nerve originates from L2-L4 and is essentially a motor nerve that innervates the medial thigh adductor muscles such as the gracilis, adductor longus brevis and magnus, pectineus and obturator externus as well as an articular branch to the knee joint. [2,3] It also gives rise to some sensory fibres to the medial thigh. Hence, an injury to the nerve can cause paraesthesia, sensory loss or pain in the medial thigh which may extend down to the knee. [2,3] It can also cause weakness in adduction with gait disturbance and medial thigh wasting which may have possible medico-legal ramifications if not managed adequately.

In this paper, we report a successful laparoscopic primary repair of an iatrogenic damaged nerve by the Harmonic Scalpel (Ethicon Endo-Surgery) during pelvic lymphadenectomy in a patient with cervical cancer.

\section{Case Report}

The patient is a 57 years old lady who was referred to the gynaecology clinic for an abnormal pap smear showing LSIL in December 2014. Colposcopy examination revealed acetowhite changes consistent with CIN 3 hence an excision biopsy was advised. She underwent a LEEP cone biopsy and unfortunately, the final histology showed an invasive squamous cell carcinoma of the cervix $(0.85 \mathrm{~cm} \times 0.3 \mathrm{~cm})$ involving the endocervical and lateral resection margins (at least pT1b). She was subsequently referred to the KK Gynaecologic Cancer Centre for further management. Clinically, there was no visible cervical tumor noted on clinical examination post LEEP. Magnetic resonance Imaging (MRI) of the abdomen and pelvis showed a small defect in the cervix in keeping with the recent LEEP and no enhancing mass was seen. There was no regional lymphadenopathy or distant metastases, hence she had a clinical Stage IB1 cervical cancer. She was counselled on various treatment options including open or laparoscopic radical hysterectomy versus primary chemoradiation. She opted for surgery and underwent a 
total laparoscopic Wertheim's radical hysterectomy, bilateral salpingo-oophorectomy and bilateral pelvic lymphadenectomy in February 2015.

During the pelvic lymphadenectomy, the left obturator nerve was inadvertently damaged and partially transected by the Harmonic scalpel about $5 \mathrm{~cm}$ from the obturator canal as it traverses under the external iliac vein. (Figure 1) After careful assessment of the damaged ends, it was noted that there was little thermal effect and a portion of the epineurium was intact hence correct orientation of the nerve for primary repair was deemed possible. The nerve was reconstituted end-to-end by three interrupted 5-O polyglactin suture to the epineural layers of the nerve. (Figure 2 and Figure 3) After that, the radical hysterectomy was completed uneventfully. Final Histology revealed a grade 3 squamous cell carcinoma $8.5 \mathrm{~mm}$ wide and $2.6 \mathrm{~mm}$ deep. There was no lymphovascular invasion nor parametrial invasion. The surgical margins was clear and $0 / 41$ pelvic lymph nodes were negative hence she did not need any adjuvant radiotherapy.

Immediate post-operatively, the patient did experience some weakness of the adductor muscles (3/5) but negligible sensory loss. Four months after intensive physiotherapy, the motor deficit was markedly reduced and her gait was normal. There was no sensory loss. She has been disease free for the last 2 years and on her last checkup in March 2017, she did not report any significant neurological impariment.

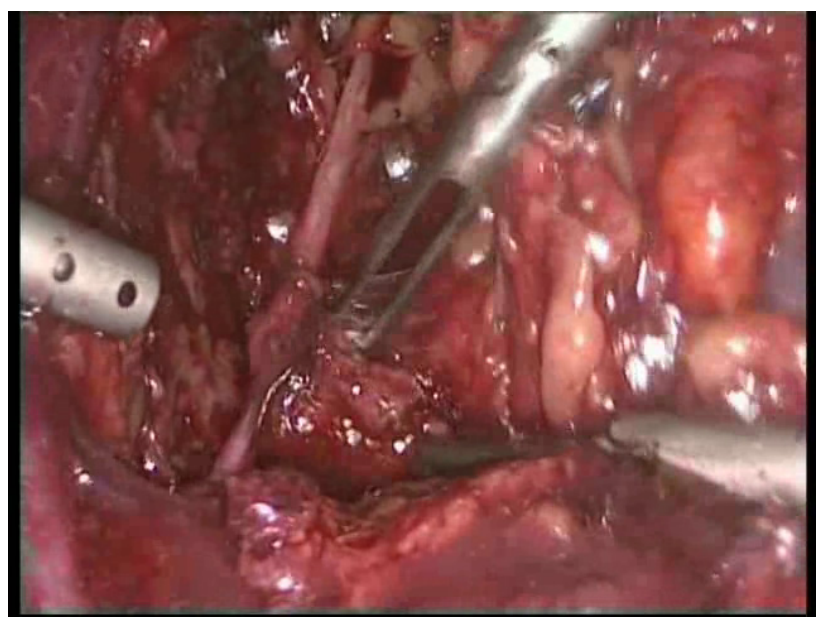

Figure 1. Near total transection of left obturator nerve

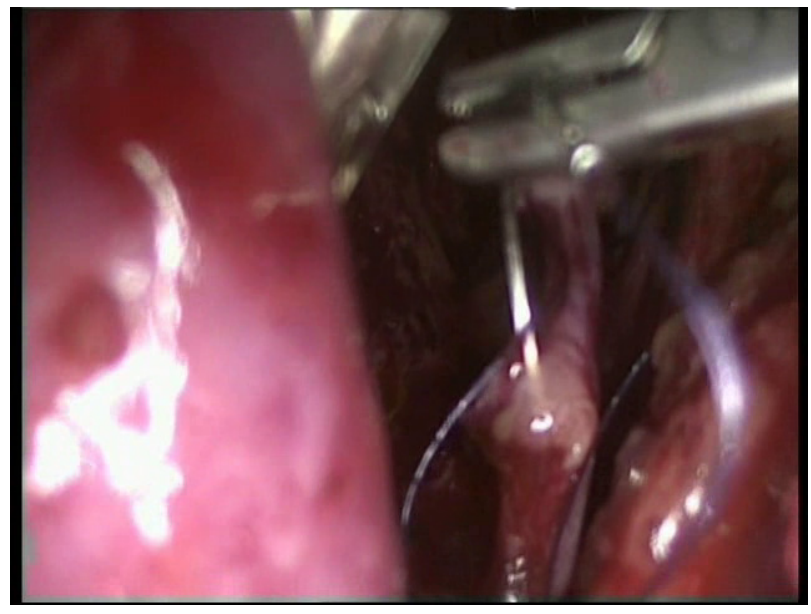

Figure 2. Laparoscopic epineural repair with polyglactin 5-O

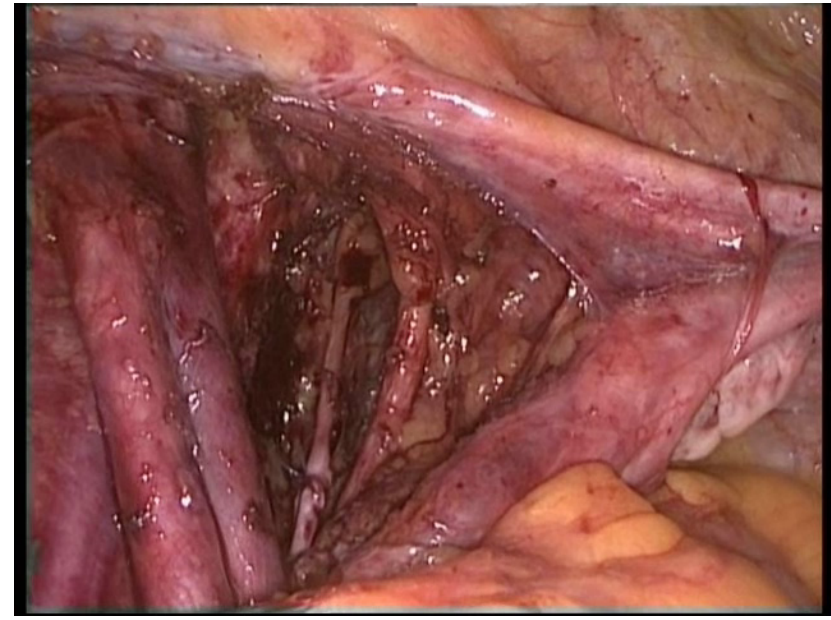

Figure 3. Final view of the repaired nerve after epineural end to end coaptation

\section{Discussion}

Injuries to the obturator nerve during complex pelvic surgery has been well documented in medical literature. The injury can occur by scissors or energy devices such as electrocoagulation, tissue sealing systems or ultrasonic devices. [4] The prognosis will depend on the nature and severity of the nerve injury. Nerve injuries can be categorised into neuropraxia, axonotmesis, and neurometsis. [2] Neuropraxia refers to a nerve contusion and is a functional injury. Axonotmesis is a more severe injury and occurs when there is degeneration of the nerve distal to the injury site even though the supporting structures remain intact. Neurotmesis is the most severe injury and refers to the complete division of the nerve. In this case, the obturator nerve was nearly totally transected with only a sliver of epineurem holding it together.

Recognition of this injury during surgery is important as an immediate repair is preferable and will usually result in functional recovery with little long-term morbidity. [5] If in doubt, an immediate neurosurgical consult is recommended. The route and type of repair will depend on the expertise available and severity of the injury. Epineural end-to-end tension free coaptation is considered the most efficient method to restore anatomy and function of the nerve using 5-O or 6-O sutures. [1,6] Alternatively, sural nerve graft interposition has been described as another method to repair especially in a situation where end to end alignment cannot be achieved or when the area of damage is too large to allow end to end anastomosis. $[4,5]$ The microsurgical repair can be achieved usually via laparotomy but there are recent reports of laparoscopic or robotic primary repair with good outcomes. $[1,6,7,8]$ The advantage for laparoscopic repair is the magnification which allows for precise repair, provided the attending surgeon can perform laparoscopic suturing.

In this case, the transection of the nerve was not complete, hence the decision was made for a primary epineural end- to end repair with vicryl 5-O. The unique feature of this case is that it shows that a near total transection of the obturator nerve with an ultrasonic device can be repaired primarily with good functional recovery. A recent case also report a similar situation 
whereby an immediate repair of an incompletely transected obturator nerve resulted in good recovery. [8] However in this case, it was during a robotic assisted pelvic lymphadenectomy for a patient with endometrial cancer.

In the postoperative phase, patients might have varying degrees of adductor muscle weakness even after a successful repair or graft. There might also be some degree of sensory loss, paraesthesia or pain in the medial thigh. [4,6] Exaggerated hip flexion should be avoided prevent tension on the suture line and physiotherapy should be implemented to strengthen voluntary adductor muscles. [2] Electromyography (EMG) can be used to ascertain the degree of obturator nerve deficit in the immediate post-operative period and the degree of recovery (reinnervation) a few months later after physiotherapy. [3] Long term follow-up is advised to ensure good functional recovery. In this case, the patient did experience some adductor weakness in the immediate post-operative period but had full neurological recovery four months after intensive physiotherapy. An EMG was not performed as the patient recovered well clinically.

\section{Conclusion}

Obturator nerve injury is a rare complication with significant clinical impact when unrecognised. An immediate repair is recommended to reduce the risk of severe neurological sequelae. In uncomplicated cases, a laparoscopic route of repair appears to be feasible and safe.

\section{References}

[1] Ricciardi E, Jakimovska M, Maniglio P, Schimberni M, Frega A, Kobal B, Moscarini M. Laparoscopic injury of the obturator nerve during fertility-sparing procedure for cervical cancer. World $\mathrm{J}$ Surg Oncol. 2012 Aug 30; 10: 177.

[2] Vasilev SA. Obturator nerve injury: a review of management options. Gynecol Oncol. 1994 May; 53(2): 152-5.

[3] Tipton JS. Obturator neuropathy. Curr Rev Musculoskelet Med. 2008 Dec; 1(3-4): 234-7.

[4] Harma M, Sel G, Açıkgöz B, Harma MI. Successful obturator nerve repairing: Intraoperative sural nerve graft harvesting in endometrium cancer patient. Int J Surg Case Rep. 2014; 5(6): 345-6.

[5] Dias AR, Silva e Silva A, Carvalho JP, Baracat EC, Favero G. Correction of iatrogenic injury of the obturator nerve during pelvic laparoscopic lymphadenectomy by the use of sural nerve grafts. Gynecol Oncol Rep. 2014 Dec 6; 10: 16-8.

[6] Nezhat FR, Chang-Jackson SC, Acholonu UC Jr, Vetere PF. Robotic-assisted laparoscopic transection and repair of an obturator nerve during pelvic lymphadenectomy for endometrial cancer. Obstet Gynecol. 2012 Feb; 119(2 Pt 2): 462-4.

[7] Menderes G, Vilardo N, Schwab CL, Azodi M.Incidental injury and repair of obturator nerve during laparoscopic pelvic lymphadenectomy. Gynecol Oncol. 2016 Jul; 142(1): 208.

[8] Göçmen A, Şanlıkan F. Immediate repair of an incompletely transected obturator nerve during robotic-assisted pelvic lymphadenectomy. J Minim Invasive Gynecol. 2015 Feb; 22(2): 302-4. 